\title{
A case of ANCA associated vasculitis presented with mononeuritis multiplex
}

\author{
Rahman Tª, Islam MR ${ }^{\mathrm{b}}$, Khan MSH${ }^{\mathrm{c}}$, Quddus SMR ${ }^{\mathrm{d}}$, Alam D ${ }^{\mathrm{e}}$, Habib R ${ }^{\mathrm{f}}$
}

\begin{abstract}
Mononeuritis multiplex is a common manifestation of many illnesses which includes diabetes, leprosy, malignancy and certain types of systemic vasculitis. The antineutrophil cytoplasmic antibody (ANCA)-associated vasculitis (AAV) is a group of rare diseases which show typical characteristic inflammatory cell infiltration and blood vessel wall necrosis. AAV syndromes include granulomatosis with polyangiitis (GPA), microscopic polyangiitis (MPA) and eosinophilicgranulomatosis with polyangiitis (EGPA). Here we present a patient who presented with mononeuritis multiplex and had features ofEGPA. The patient was treated with standard regimen of steroids and pulsed cyclophosphamide and she achieved good clinical response.
\end{abstract}

Keywords: ANCA associated vasculitis, eosinophilic granulomatosis with polyangiitis, microscopic polyangiitis, granulomatosis with polyangiitis.

(BIRDEM Med J 2019; 9(3): 257-260)

\section{Introduction}

The ANCA-associated vasculitis (AAV) is a group of rare diseases showing characteristic inflammatory cell infiltration and blood vessel wall necrosis in histology. AAV is generally used to include primary vasculitis syndromes in which circulating ANCA against proteinase 3 (PR3) called c-ANCA and myelo-

Author information

a. Dr. Tanbin Rahman,MBBS, MRCP(UK), Registrar, Medicine, Greenlife Medical College and Hospital, Dhaka, Bangladesh.

b. Dr. Md. Rashedul Islam, MBBS, FCPS (Neurology),MRCP (UK), FCPS (Medicine), FACP (USA), Assistant Professor, Department of Neurology, BIRDEM General Hospital, Dhaka, Bangladesh.

c. Dr. Mohammad Sakhawat Hossen Khan, MBBS, FCPS (Medicine), MRCP(UK), Registrar, Department of Neurology, BIRDEM General Hospital, Dhaka, Bangladesh.

d. Dr. Sharif Mohammad Ruhul Quddus, MBBS, MRCP(UK), Assistant Registrar, Department of Neurology, BIRDEM General Hospital, Dhaka, Bangladesh.

e. Dr. Dilruba Alam, MBBS, FCPS (Medicine), Registrar, Department of Neurology, BIRDEM General Hospital, Dhaka, Bangladesh.

f. Dr. Rumana Habib, MBBS, FCPS (Medicine), Associate Professor, Department of Neurology, BIRDEM General Hospital, Dhaka, Bangladesh.

Address of correspondence: Dr. Tanbin Rahman, Registrar, Department of Medicine, Green Life Medical College and Hospital ,Dhaka, Bangladesh. Email: tanbinrahman@yahoo.com

Received: August 7, 2019

Accepted: August 10, 2019 peroxidase (MPO) called p-ANCA are commonly found.AAV syndromes include granulomatosis with polyangiitis (GPA), microscopic polyangiitis (MPA) and eosinophilic granulomatosis with polyangiitis (EGPA). ${ }^{1}$

There is no definitive classification of systemic vasculitis because of its varied aetiology which is largely unknown. There is considerable overlap in the clinical presentation of the different vasculitic syndromes. The general way of classification is based on the size of dominant vessel and ANCA status. However, AAV is grouped separately since they involve small and medium-sized arteries which are frequently ANCA associated. Also, AAV is associated with increased risk of glomerulonephritis and respond adequately to immunosupression (cyclophosphamide). ${ }^{2}$ The probable reason is that the aetiology may not be related to immune complex formation unlike pure small-vessel vasculitis like Henoch-Schönlein purpura and cryoglobulinaemic vasculitis. In 1990, the American College of Rheumatology (ACR) developed classification criteria for systemic vasculitis. They did not consider microscopic polyangiitis but considered Wegener's granulomatosis, Churg-Strauss syndrome and polyarteritisnodosa. The Chapel Hill Consensus Conference (CHCC) in 1994 defined AAV which comprised microscopic polyangiitis but lacked the 
criteria to diagnose. ${ }^{3,4}$ These were revised and updated in 2012 and a revised nomenclature introduced granulomatosis with polyangiitis (Wegener's granulomatosis) (GPA), MPA, and EGPA (ChurgStrauss syndrome).$^{5}$

\section{Case report}

A 60-year-old non diabetic, hypertensive lady presented with weakness of left leg initially then it affected right leg for the last 1 month. Weakness was gradual in onset, progressive and eventually caused walking difficulty. Subsequently it involved her upper limbs also for the last 2 weeks. On query, she had complaints of arthralgia and numbness which was continuous. Numbness was prominent on fingers and toes, more marked at night. She also developed skin rash for the last 14 days involving palm, sole, feet and forearm which was painful, non palpable, symmetrically distributed. There was no history of fever, joint swelling, morning stiffness, weight loss, mouth ulcer, alopecia, color change of hands on exposure to cold. She has history of late onset asthma which was diagnosed 5 years back and she is on regular treatment. She is non smoker, non alcoholic with no history of substance misuse. She had no family history of any significant illness. She was on antihypertensive medication.

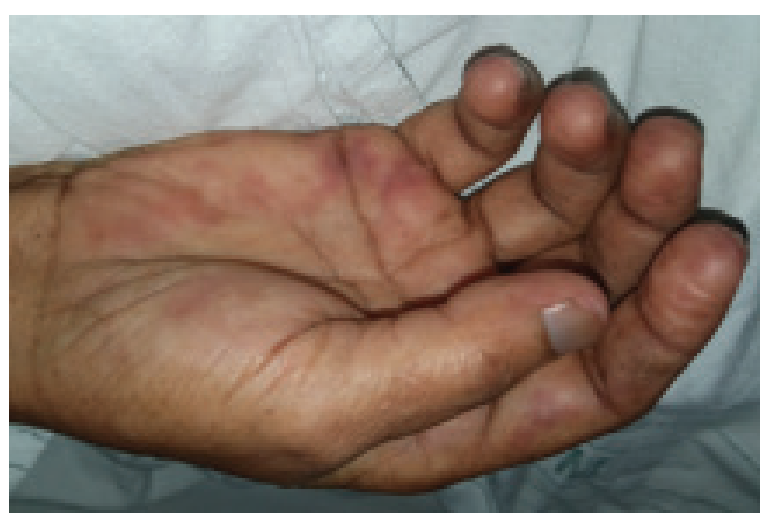

Figure 1 Rash on palmar surface of hand

On examination, she was anxious, ill looking, and anemic. She had purplish non palpable and painful rash involving palm, sole, forearm and feet. (Fig-1) Vital signs were normal including blood pressure. On neurological examination; she had normal higher psychic function including speech. She also had generalized hypotonia, bilateral foot and wrist drop (Figure 2), diminished deep tendon reflexes and high steppage gait.(Fig-2) All modalities of sensation were diminished in gloves and stocking pattern. Other systemic examination revealed no abnormalities.

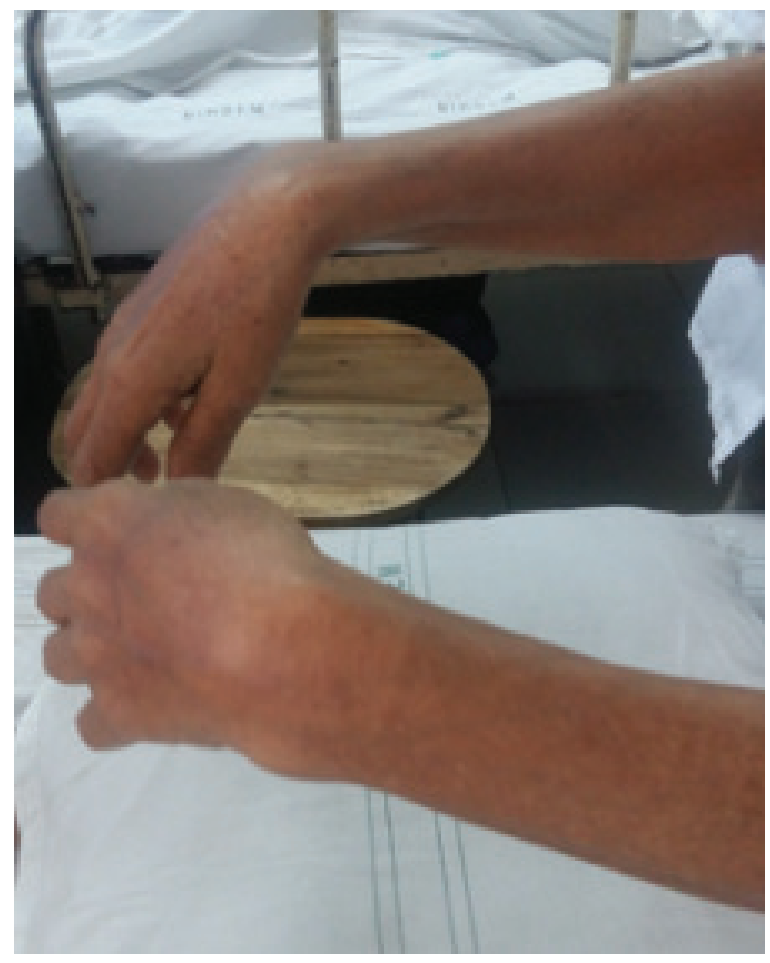

Figure 2 Bilateral wrist drop

On investigation, she had anemia ( $\mathrm{Hb} \%-10.4 \mathrm{~g} / \mathrm{dl})$, eosinophilia (16\%), raised ESR (69 $\mathrm{mm}$ in first hour). $\mathrm{C}$ - reactive protein (CRP) and perinuclear antineutrophil cytoplasmic antibody (p-ANCA) was positive. Her routine investigations including liver and renal function test, electrolytes and urine examination were within normal limits. Serologic tests for Hepatitis B, Hepatitis C were negative. Anti-Nuclear Antibody (ANA) was negative. Chest radiograph and electrocardiogram, X-ray paranasalsinus revealed no abnormality. Nerve Conduction Study (NCS) revealed low amplitude compound muscle action potential (CMAP) in common peroneal nerve, tibial nerve, median nerve, ulnar nerve, radial nerve and absent sural nerve, median nerve, ulnar nerve, radial nerve sensory nerve action potential (SNAPs) bilaterally. Electromyogram(EMG) showed neuropathic motor unit potential. Sural nerve biopsy showed degenerating nerve 
fiber and endoneural infiltration of inflammatory cell (polymorphs, eosinophil and lymphocytes) in and around the neural blood vessel suggestive of vasculiticneuropathy (fig-3). Doppler study of both lower limbs showed mild atherosclerotic change. We treated the patient with Inj Methylprednisolone (1 gm IV infusion/day for five days) and cyclophosphamide pulse therapy ( $1 \mathrm{gm} / \mathrm{m}^{2} \mathrm{IV}$ every month). She had shown slow improvement. We continued two monthly cyclophosphamide pulses after first six months based on good therapeutic response and have plan to continue for one year. She will be followed up thereafter.

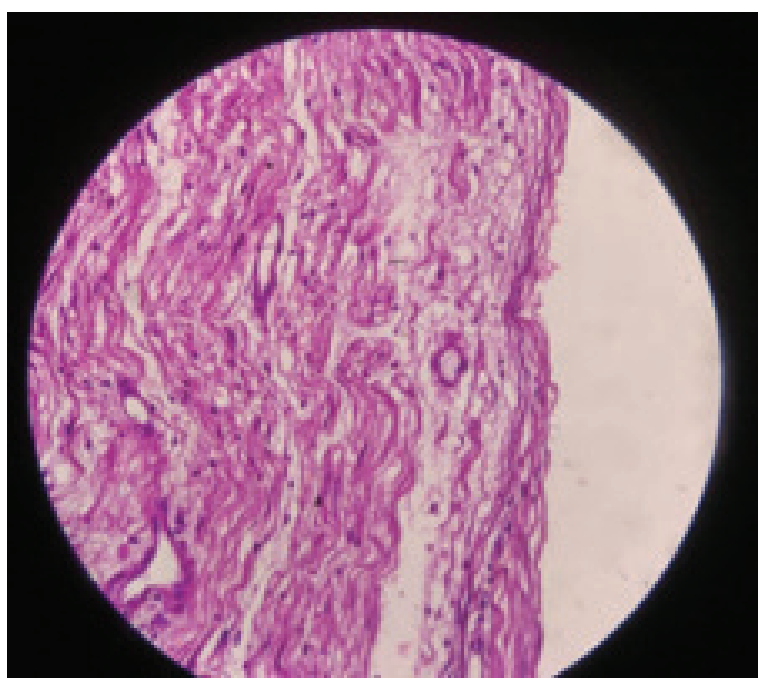

Figure 3 Sural nerve biopsy showingdegenerating nerve fiber and endoneural infiltration of inflammatory cell in and around the neural blood vessel

\section{Discussion}

GPA previously known as Wegener granulomatosis, is an uncommon autoimmune disease. The disease spectrum includes necrotising granulomata of the respiratory tract (upper and lower), necrotisingvasculitis involving various organs like eyes, throat, skin and frequently involves the kidneys causing focal glomerulonephritis. The most common clinical presentation is the upper airway disease. It is seen in upto $90 \%$ of patients and almost $70 \%$ of patients initially present with upper airway disease. ${ }^{6}$ Destruction of the nasal septum results in the typical saddle-nose deformity. About $85 \%$ of patients develop sinusitis during the course of the disease. Involvement of larynx and trachea is usually asymptomatic, but may present with hoarseness or stridor. ${ }^{6}$
Neurological involvement is uncommon at presentation and presents as peripheral neuropathy or mononeuritis multiplex. Mostly the symptoms are subclinical and can be detected in d" $50 \%$ of patients in the course of illness. ${ }^{7}$

The initial description of MPA was given by Kussmaul and Maier when they described a patient showing periarteritis with inflammatory necrosis of medium-sized arteries resulting in organ infarction. The term microscopic polyarteritis nodosa was first described by Wainwright and Davson in a group of patients who in addition to features of PolyarteritisNodosa (PAN) also had segmental glomerulonephritis manifesting as rapidly progressive renal failure. ${ }^{8}$

Renal involvement is characteristic for $\mathrm{MPA}^{8}$. The occurrence of Mononeuritis multiplex in MPA is uncommon $(\leq 20 \%)$ similar to GPA. Also, eye and nasopharyngeal involvement are uncommon in MPA than in GPA $(<30 \%){ }^{6}$

The characteristic clinical features of EGPA include asthma which typically occurs late in the course of the disease associated with peripheral blood and tissue eosinophilia alongwith fever and systemic vasculitis. ${ }^{9}$ The occurrence of asthma which precedes the onset of systemic features in almost all cases of EGPA distinguishes it from other forms of vasculitis. ${ }^{9}$ Unlike MPA and GPA, mononeuritis multiplex is a common $(70 \%)$ feature of EGPA. ${ }^{10}$

Our patient had late onset bronchial asthma for which she was under regular medication. She had mononeuritis multiplex involving the upper and lower limbs which was gradually progressive. There was evidence of peripheral eosinophilia. Nerve biopsy was suggestive of vasculitis with eosinophilic infiltrate. The clinical picture, evaluation and investigation are suggestive of EGPA. Also, there is no renal involvement to suggest MPA. The absence of respiratory tract and renal involvement, rules out GPA as a possibility.

Hence, our patient is most likely suffering from EGPAand the patient showed good response with steroids and pulse cyclophosphamide therapy.

\section{Conclusion}

ANCA-associated vasculitis is an uncommon disease entity. Early diagnosis and prompt treatment can ensure better outcome for the patient. Our patient showed good clinical response and now independent for all activities of daily living.

Conflict of interest: Nothing to declare. 


\section{References}

1. Xiao H, Hu P, Falk R, Jennette J. Overview of the pathogenesis of ANCA-associated vasculitis. Kidney Diseases. 2015; 1(4):205-15.

2. Harper L, Morgan M, Walsh M, Hoglund P, Westman K, Flossmann O, et al. Pulse versus daily oral cyclophosphamide for induction of remission in ANCA-associated vasculitis: Long-term follow-up. Annals of the Rheumatic Diseases. 2011; 71(6):955-60.

3. Fries J, Hunder G, Bloch D, Michel B, Arend W, Calabrese L, et al. The American College of Rheumatology 1990 criteria for the classification of vasculitis: Summary. Arthritis \& Rheumatism. 1990; 33(8):1135-36.

4. Jennette J, Falk R, Andrassy K, Bacon P, Churg J, Gross W, et al. Nomenclature of Systemic Vasculitides. Arthritis \& Rheumatism. 1994; 37(2):187-92.

5. Jennette J, Falk R, Bacon P, Basu N, Cid M, Ferrario F, et al. 2012 Revised International Chapel Hill Consensus Conference Nomenclature of Vasculitides. Arthritis \& Rheumatism. 2012; 65(1):1-11.
6. Harabuchi Y, Kishibe K, Komabayashi Y. Clinical manifestations of granulomatosis with polyangiitis (Wegener's granulomatosis) in the upper respiratory tract seen by otolaryngologists in Japan. ClinExpNephrol. 2013; 17(5):663-66.

7. Langford CA, Fauci AS.The Vasculitis Syndromes.In: Kasper DL, Fauci AS, Hauser, Longo, Jameson, Loscalzo, editors. Harrison's principles of internal medicine, 19thEd. New York (NY): The McGraw-Hill Companies, Inc; 2015. pp. 2179 2193.

8. Villiger P, Guillevin L. Microscopic polyangiitis: Clinical presentation. Autoimmunity Reviews. 2010; 9(12):812-19.

9. Gioffredi A, Maritati F, Oliva E, Buzio C. Eosinophilicgranulomatosis with polyangiitis: An overview. Front Immunol. 2014; 5:549.

10. Uematsu H, Takata S, Sueishi K, Inoue, H. Polyangiitis overlap syndrome of granulomatosis with polyangiitis (Wegener's granulomatosis) and eosinophilicgranulomatosis with polyangiitis (Churg-Strauss syndrome) BMJ Case Reports. 2014; 2014:bcr2013010195. 